\title{
De Ofertas y Demandas: Una Propuesta de Intervención en Psicología Comunitaria
}

\section{On Supply and Demand: An Intervention Proposal in Community Psychology}

\author{
Psic. Alicia Rodríguez (aliciar@psico.edu.uy)* \\ Psic. Luis Giménez (luisg@psico.edu.uy)* \\ Lic. Clara Netto (cnetto@psico.edu.uy)* \\ Psic. María José Bagnato (majose@psico.edu.uy)* \\ Lic. Cecilia Marotta*
}

\section{Resumen}

El artículo parte de la experiencia educativa que los autores desarrollan como docentes del curso Técnicas de Atención Comunitaria de la Facultad de Psicología-Universidad de la República, de Montevideo,Uruguay. Del análisis de la práctica y de la constatación de frecuentes confusiones conceptuales, surge la importancia de delimitar y dilucidar las nociones de necesidad, pedido, demanda y encargo, para la especificidad del trabajo comunitario. Ubicándose en el inicio de una intervención y reconociendo el carácter fundante de ese momento, enfatizan la interacción entre la construcción de la demanda y la oferta, proceso que se desencadena en presencia o no de un pedido explícito por parte de la comunidad. Explicitan algunas interrogantes sobre la implicación de la Psicología Comunitaria en el contexto social actual.

Palabras Claves: Intervención comunitaria. Necesidades. Demanda.

\section{Abstract}

The authors share an educational experience as professors of the course Techniques in Community Service at the School of Psychology, University of the Republic, Montevideo, Uruguay.

Practice analysis and evidence of frequent concept misunderstandings show the importance to elucidate and clarify notions such as 'need', 'order', 'demand' and 'assignment' in the frame of community work.

Placing themselves at the beginning of an intervention, and admitting its founding nature, they emphasize interaction in the building of demand and supply as a process that evolves in the presence, or not, of an explicit order on the side of community. They state some questions about community psychology implication in the present social context.

\footnotetext{
* Equipo docente del curso Técnicas de Atención Comunitaria del Area de Salud de la Facultad de Psicología de la Universidad de la República.Montevideo, Uruguay
} 


\section{Introducción}

Los autores del presente trabajo conformamos un equipo docente que desarrolla un curso llamado "Técnicas de Atención Comunitaria" en el Area de Salud de la Facultad de Psicología de la Universidad de la República en Montevideo, Uruguay.

Se trata de un curso relativamente joven (el primero dictado en 1994) que se implementa en el último año de la formación de los psicólogos y su propósito es brindar al estudiante elementos teóricos, metodológicos y técnicos de Psicología Comunitaria para su futuro desempeño profesional.

En estos años, y por distintos caminos, fuimos llegando a la necesidad de profundizar en algunos conceptos claves vinculados a los momentos fundantes de la Intervención Comunitaria. Esos conceptos son: necesidades, pedido, demanda y encargo.

Para los estudiantes, en el contexto de sus prácticas universitarias, el tema de la demanda se les presenta muchas veces como un obstáculo para procesar su inserción en la comunidad. Perciben que los roles quedan invertidos: ellos en el lugar de quienes demandan un ámbito específico en donde desarrollar su pasantía en el marco de su formación profesional, y la comunidad como la que está dispuesta a "brindarles un servicio" al otorgarles esa posibilidad. "No hay demanda", suelen decir.

La preocupación de los estudiantes es legítima, si la consideramos como el rechazo al establecimiento de un vínculo de carácter utilitario con la comunidad, en donde se extraen cosas (información, conocimientos) cayendo en un uso y abuso de la misma sin dejar nada a cambio. Se trata pues, de una saludable preocupación de orden ético.

Pero creemos que también subyacen otros aspectos.
Por un lado, la idea de que se establece un vínculo asimétrico entre el técnico y la comunidad en donde uno queda en el lugar de dar y el otro en el de recibir, en vez de concebir la relación como un intercambio en el que se ponen en juego necesidades y expectativas recíprocas.

Por otra parte, la llamada demanda, tiende a aislarse de la oferta y a ser cosificada y tratada como un objeto, que puede estar o no, y que como tal, se supone que preexiste a la presencia del profesional.

Se agrega además que los estudiantes llegan al último año con un código incorporado que incluye una o más formas de entender las intervenciones psicológicas, la relación entre el técnico y aquellos a quienes va dirigido su trabajo, las formas de producción de los fenómenos sociales y del conocimiento acerca de los mismos.

Si bien desde su ingreso a Facultad, reciben una panorámica relativamente amplia en relación a las diversas corrientes disciplinarias, a las posibilidades de inserción del psicólogo y a los modelos profesionales concomitantes, tanto ellos como nosotros y la comunidad toda, somos tributarios de un modelo médico-hegemónico funcional a una lectura del psicólogo haciéndose cargo de lo enfermo desde un ámbito clínico. A ello se suma una fuerte impronta psicoanalítica propia del desarrollo de la psicología en el Rio de la Plata.

Es mucho lo que el Psicoanálisis nos ha aportado a nuestra formación profesional y particularmente a la comprensión de los fenómenos psicológicos, pero es riesgoso intentar trasladar a un plano comunitario nociones que fueron concebidas para lo intrapsíquico.

Partiendo de estos elementos, entonces, es que nos planteamos la necesidad de reflexionar acerca de la temática de la Demanda en la relación técnico-comunidad desde la particular perspectiva de la Psicología Comunitaria. 


\section{Elementos a Tener en Cuenta al} Implementar una Intervención

\section{Comunitaria}

El momento inicial de una intervención, en tanto fundante, pautará el desarrollo de la misma. La ausencia de análisis en esta etapa conlleva el riesgo de un hacer irreflexivo en donde la direccionalidad escapa a la intencionalidad de los actores en juego.

El contrato de trabajo con los destinatarios supone esa reflexión previa que posibilitará lo que A. Lapalma (1990) llama el «establecimiento de la relación inicial» rescatando el concepto que Lippit utiliza para el ámbito organizacional. Permite un ajuste de expectativas a la vez que obliga a recortar el campo de trabajo. En este sentido, implica dejar cosas afuera, tomar conciencia de los límites y llevar la intervención al campo de lo posible.

Las intervenciones comunitarias pueden fundarse de diversas formas:

a - A partir de un pedido formulado directamente por la comunidad, a través de alguno o algunos de sus portavoces. Perciben la insatisfacción de determinadas necesidades o la presencia de problemas para cuyo abordaje piden ayuda.

b - A partir de un pedido formulado por un tercero (técnico, organización, etc.). Puede suceder que la comunidad perciba sus necesidades y problemas pero no sepa a quién recurrir y entonces el tercero oficia de «puente». O bien, éste valora la necesidad de una intervención psicológica, a partir de una lectura propia de los problemas del colectivo.

c - A partir de una oferta. Cuando no aparece un pedido y la intervención se genera desde la oferta de un servicio, ya sea de un psicólogo que presenta un proyecto o de una organización que desde sus objetivos se plantea la acción en determinada zona o con determinada población.

En todos estos casos, la estrategia será distinta pero siempre supondrá transitar por la construcción de un vínculo para posibilitar el desarrollo de un trabajo.

\section{Conceptos Claves}

Para posibilitar el análisis del momento inicial de la intervención hay cuatro términos, que a nuestro criterio deben ser abordados, tanto en la especificidad de cada uno de ellos, como en su interrelación. Ellos son: necesidades, pedido, demanda y encargo.

\section{Necesidades}

De las nociones antes mencionadas, la de necesidad es la de uso más frecuente en lo comunitario.

El proceso de identificación de necesidades por parte de los propios actores comunitarios resulta un momento clave para la explicitación de los problemas a abordar. Lo cual supone: percibir las necesidades comunes, descubrir su significado y ordenarlas jerárquicamente.

M.Montero (1991) plantea que según cual sea su origen perceptivo las necesidades pueden clasificarse en normativas o inferidas y sentidas. En el primer caso, las necesidades surgen de la comparación que un agente externo realiza entre la realidad y un canon pre-establecido. En el caso de las necesidades sentidas son las propias personas que las manifiestan explícita o implícitamente.

Es usual que en el diseño de políticas sociales y de salud, se priorice determinadas necesidades definidas desde criterios de experticia, con lo cual se vehiculiza concep- 
ciones propias de quienes las formulan, $\mathrm{mu}-$ chas veces alejadas de lo que desde los involucrados se valora como problema.

Desde la perspectiva de la Psicología Comunitaria la cuestión no pasa por la «verdad» de las necesidades así definidas, sino por su condición de existencia para los que en definitiva serán sujetos de la intervención. La acción comunitaria requiere la expresión de una necesidad, y ésta es resultado de un proceso que, partiendo del sentir incorpora elementos de concientización que permitan verla, es decir darle existencia. El trabajo del psicólogo pasa en esta etapa por facilitar el intercambio y la problematización que genere las condiciones para identificar colectivamente las necesidades (Montero, 1991).

En este campo el aporte de Manfred Max Neef (1986) nos ha resultado pertinente. Frente a las reiteradas confusiones que el tema genera plantea la distinción entre necesidades y satisfactores. De tal manera que, mientras las necesidades serían universales, de número limitado y clasificables, los satisfactores serían objeto de múltiples variaciones históricas y culturales. Este autor ordena las necesidades según criterios existenciales (ser, tener, hacer y estar), y axiológicos (subsistencia, protección, afecto, entendimiento, participación, ocio, creación, identidad, libertad). La matriz de necesidades y satisfactores conformada, constituye una interesante herramienta diagnóstica para trabajar junto con los diferentes sujetos comunitarios en la delimitación de los problemas y sus posibles alternativas.

Los satisfactores no actúan colmando las necesidades sino que constituyen los modos culturalmente establecidos para actualizarlas de forma continua y renovada. Existen diferentes tipos de satisfactores y no todos posibilitan vivir las necesidades con igual intensidad y plenitud. La propia intervención que como agentes externos llevamos adelante, puede ser pensada como un satisfactor que actúa frente a ciertas necesidades. Es así que, el aporte de Max Neef, contribuye a la evaluación y la imprescindible autocrítica y reflexión que debe acompañar toda intervención comunitaria.

\section{Pedido}

La autopercepción de las necesidades supone que las personas nos podamos reconocer como carentes. Ello incluye la desilusión y la ruptura de la omnipotencia, el reconocimiento de los límites. Pero a la vez, y tal como lo expresa Max-Neef (1986) la necesidad es potencialidad en tanto se constituye en un motor que nos pone en movimiento para buscar su satisfacción y para pedir ayuda cuando la misma se hace necesaria. La explicitación del deseo de satisfacer las necesidades y de resolver los problemas con la ayuda de un otro, se constituye en la formulación de un pedido. El pedido es entonces la explicitación de la necesidad de ayuda, dirigida a quien o quienes se cree podrán responder a la misma.

\section{Demanda. Proceso de construcción de la demanda}

A partir de la explicitación del pedido, será necesario entonces abrir el juego a la construcción de la demanda.

La demanda no puede ser entendida sin considerar su relación con la oferta. Es sólo desde esta última, que la primera se puede desplegar. Dicho interjuego está siempre presente, exista o no un pedido de intervención.

Cuando existe un pedido explícito, el mismo se genera desde la idea de que alguien puede darle respuesta. Dicho pedido merece una lectura y una problematización en el seno del vínculo entre el técnico y quien o quienes lo formulan. Se producirá allí algo novedoso para ambos. Ese pedido, como decíamos antes, podrá provenir directamente de la comunidad o bien, podrá ser formula- 
do por un tercero. En ambos casos la estrategia será distinta pero siempre supondrá transitar por la construcción de un vínculo para posibilitar el desarrollo de un trabajo.

Cuando no aparece un pedido, y la intervención se genera desde la oferta de un servicio, se abre la posibilidad a la demanda. La propia oferta y la eventualidad de que ella sea aceptada, implica un cierto diagnóstico de situación y de coyuntura. De modo que su formulación, si es pertinente, se articulará con las necesidades de los destinatarios. Desde allí entonces, la demanda puede ser generada y desplegarse como tal.

Nos referimos a una construcción entre ambos términos de la relación. Compartimos con la perspectiva del Construccionismo Social la noción de que la realidad se construye socialmente. De que si bien preexiste a la presencia del técnico y los fenómenos no pueden ser entendidos si no es históricamente, cuando el agente externo entra en contacto con la misma, se genera un campo intersubjetivo que modifica a los actores en juego y genera nuevos procesos determinados por esa relación. Dicha construcción puede tener sentidos diferentes en las situaciones antes descritas.

\section{La Problematización del Pedido}

La lectura del pedido cuando existe, incluye su problematización y un análisis de los aspectos implícitos. Se trata de dilucidar cuál es ese pedido y cómo posicionarse ante el mismo. Se trata de formular colectivamente una serie de interrogantes: ¿qué significado tiene este pedido en la situación actual de quienes lo formulan?, ¿cómo se inscribe en la historia del colectivo?, ¿quién o quiénes la formulan y por qué es él o ellos? ¿qué piensan los demás integrantes del colectivo sobre este pedido? ¿qué valor de emergente podemos otorgar al mismo?, ¿por qué a nosotros?, ¿qué expectativa se depositan en nuestra intervención?. La respuesta a éstas y a otras interrogantes deberá surgir de la relación misma entre el técnico y la comunidad.

Problematizar supone entonces, abrir un espacio para la comprensión, romper con la mirada ingenua y con la idea de que la realidad es una y podemos ir a su encuentro mediante un camino lineal. Problematizar supone abrir el espacio para la complejidad y para la dinámica de la realidad, rompiendo sin embargo con la fantasía omnipotente de poder aprehenderla.

Este momento de la intervención es un momento de apertura, tendiente a posibilitar el desarrollo de un trabajo que genere algún tipo de cambio en la situación planteada y el sentido de dicho cambio surgirá de este encuentro. La respuesta directa al pedido y sin cuestionamiento alguno, puede implicar un cierre a dichas posibilidades.

Sin embargo, no siempre están dadas las condiciones para dar lugar a un intercambio y problematización del pedido. En ocasiones, responder directamente a lo que se pide, se constituye en una táctica de trabajo que facilita la construcción de un vínculo de confianza y abre la posibilidad de que la palabra del técnico sea escuchada. La reflexión conjunta en torno al pedido, podrá realizarse en un momento posterior, a veces como «cierre» de la intervención. En algunos casos, instrumentar los cursos o los talleres que se nos piden, o atender un problema individual en el ámbito del consultorio ,o responder a la maestra sobre sus inquietudes en relación a los problemas de aprendizaje que presenta un niño, se constituye en la respuesta pertinente. Ello no significa que no abramos la posibilidad a una reflexión que posibilite dar un mayor alcance a nuestra intervención. 
Las condiciones para interrogar directamente el pedido pueden no estar dadas por diferentes razones. Por un lado el tiempo real con el que se cuenta, puede requerir que el análisis del pedido lo realicemos en forma simultánea a la ejecución de la propuesta. Tal es el caso de aquellas intervenciones que «vienen planificadas» por quienes las organizan e incluso con días y horas ya establecidos. Tomamos contacto con los destinatarios directos en el momento mismo de la implementación de la propuesta. Por lo general consisten en unas pocas jornadas de trabajo. En algunos casos, ello permitirá realizar modificaciones en la marcha. Generará la posibilidad de aprendizajes para la instrumentación de nuevas propuestas que podrán ser compartidas con los destinatarios directos y con los organizadores de la actividad.

Otras veces, las «urgencias» que nos plantea el colectivo con el que vamos a trabajar, determinan la necesidad de respuestas más o menos inmediatas y los tiempos para la reflexión vienen a posteriori. No obstante, es importante distinguir entre los apremios de cada situación (que muchas veces son tales si consideramos las situaciones límite a las que nos vemos enfrentados) y la tendencia riesgosa a actuar sin reflexionar ante circunstancias que nos generan una movilización afectiva importante. La estructura de demora es fundamental, y sabemos que la misma alude más bien a una actitud psicológica que a los tiempos que marca el calendario.

También podemos encontrarnos con que las condiciones para la problematización del pedido, no están dadas por los tiempos internos del colectivo. No debemos olvidar que la realidad es un campo de fuerzas en donde las resistencias al cambio están siempre presentes. Será necesario buscar las formas y los espacios en donde las transformaciones puedan tener lugar.

En síntesis, una actitud estratégica que permita valorar a cada momento los límites y posibilidades, los obstáculos y los facilitadores, será fundamental para poner los objetivos de la intervención al alcance.

\section{Partiendo desde la Oferta de Intervención}

En el caso en que el pedido no se formula desde la comunidad, o desde un tercero, sino que lo que abre la intervención es una oferta, el proceso puede darse de diferente forma:

A partir de un diagnóstico en un sentido amplio, que implica un análisis de coyuntura, se construye una propuesta que tendrá que ser puesta a prueba en la realidad. Hay aquí un componente creativo que se apoya en un saber acumulado a partir de otras experiencias, lo cual «garantiza» en cierta medida la pertinencia de la propuesta, pero puede ser un elemento que obture si no se adecua a cada momento y a cada situación.

Este movimiento tiene que ver con el análisis de una demanda social que estará determinada tanto por variables económicas y políticas, como socio-culturales. Así, por ejemplo, los fenómenos de desafiliación y de deterioro de las redes de sostén a los que asistimos en la sociedad actual, como efecto de la persistencia de un modelo neoliberal, no pueden dejar de considerarse en la formulación de los proyectos comunitarios que pretendamos implementar.

O puede partir de un diagnóstico acotado a una situación particular, que permita determinar la pertinencia de la propuesta de trabajo con determinado sector de la población. Requerirá por parte del psicólogo una serie de acciones tendientes a involucrar en la propuesta a los actores de la comunidad. Y en ese proceso deberá darse lugar a la flexibilidad necesaria para la introducción de los cambios que la situación requiera. De no 
existir articulación con las necesidades del otro la intervención no será posible. En esa articulación es que se va construyendo la demanda, lo cual requerirá de un proceso de análisis permanente.

\section{Construcción de Demanda y Diversidad}

Frecuentemente hablamos de la comunidad y la demanda de la comunidad, con lo cual otra vez ponemos en evidencia nuestra tendencia a considerar la realidad como un algo homogéneo y exento de diversidad y conflicto. Es esencial a la misma el aspecto de diversidad y por lo tanto, cuando hablamos de construcción, y de construcción colectiva, aludimos a la apertura necesaria a dicha diversidad. Interrogarnos acerca de qué piensan otros sobre el pedido implica dar lugar a las diferentes opiniones, necesidades, expectativas, priorizaciones, percepciones y por tanto a diferentes pedidos. Implica dar lugar al conflicto producto de las luchas por el poder, presente en toda relación humana. En definitiva, implica posibilitar el despliegue de las distintas subjetividades presentes respecto a una misma realidad «objetiva». La construcción de la demanda no será pues, una sumatoria de los pedidos formulados, sino más bien el producto del interjuego de las diversas subjetividades.

\section{Encargo}

La formulación del pedido lleva consigo una serie de depositaciones en el psicólogo. A ello nos referimos cuando hablamos de encargo. Depositaciones que implican el reconocimiento de un saber y de una autoridad en el profesional y que por tanto posibilitan una intervención. Y también, depositaciones que obstaculizan, en la medida que significa poner en manos de otro la resolución de la situación, sin dar lugar a un intercambio donde ambos términos de la relación tengan un papel activo y protagónico.

Será imprescindible entonces, aceptar una parte de la depositación para poder trabajar, aquélla que nos legitima en el lugar de poder brindar algún tipo de ayuda a quienes la necesitan. Muchas veces se cae en una actitud de «devolver» a la comunidad, aspectos de dicha depositación en un momento no oportuno, aduciendo la promoción de la autogestión, cuando en realidad lo que está sucediendo es que el técnico no esta pudiendo asumir una parte de lo depositado para retrabajarlo en un proceso analítico. Se genera así un efecto «rebote» que no permite sostener o construir el vínculo. Creemos que hace a una actitud ética, en tanto se trata de hacernos cargo de procurar una ayuda y de poner nuestros conocimientos y herramientas de intervención al servicio de quienes nos requieren.

El otro extremo es hacerse cargo de la depositación en su totalidad, asumiendo un lugar de satisfactor-inhibidor en tanto se concentra todo el poder en una parte de la relación. Lo percibimos cuando asumimos los roles que se nos depositan sin poder pensar, roles que muchas veces tienen que ver con lo que no puede ser hablado (el lugar del juez, o el de estirpar lo enfermo, el de la denuncia, etc.).

Estos aspectos suponen ponerse en juego uno mismo en el diagnóstico de situación, analizar nuestra implicación, reconocernos también como sujetos de necesidades, que como el resto, las busca satisfacer en las relaciones que establece. Suponen pensarnos como representantes de diversas instituciones, la del mundo académico, la de la psicología, la de la intelectualidad y la de la clase media uruguaya, entre otras. Supone pensarnos como representantes de las organizaciones que integramos y desde las cuales actuamos. Las expectativas en relación a 
nuestra intervención estarán "contaminadas» 0 «condimentadas» del lugar social que le sea otorgado a las organizaciones desde las que actuamos. Suponen en definitiva, reflexionar sobre nuestro posicionamiento en la intervención, el que resultará del entrecruzamiento de una historia personal y social, que incluye valores, sentimientos y una particular percepción de la realidad.

\section{Conclusiones}

Podríamos tomar los conceptos antes desarrollados ya no sólo para pensar las múltiples intervenciones comunitarias que llevamos adelante, sino también para reflexionar sobre el papel de la Psicología Comunitaria en los tiempos que corren.

Como disciplina o como un particular enfoque de la psicología, la Psicología Comunitaria se constituye en una oferta que busca dar respuesta a los problemas sociales que hoy nos aquejan.

Es desde allí que nos formulamos preguntas tales como:

¿Cuál es el lugar de la Psicología Comunitaria en la realidad social actual y en el escenario particular de la Psicología? ¿Qué necesidades están planteadas? ¿Qué demandas se van construyendo? ¿Cuál es el encargo hacia los psicólogos?

El contexto social en el que convivimos, producto de la aplicación de un modelo neoliberal que trasciende lo estrictamente económico para «filtrarse» en la cotidianeidad de las relaciones interpersonales y de la subjetividad, ha venido generando nuevos fenómenos de exclusión y desafiliación en las redes sociales.
¿Cómo intervenir en los procesos de búsqueda de sentido de una comunidad que asiste a la caída de instituciones tales como el trabajo, los sindicatos, las tradicionales formas de participación, la familia nuclear, etc? ¿Al desarrollo tecnológico que posibilita nuevas e inimaginadas formas de comunicación? ¿Qué funcionalidad tendríamos los psicólogos comunitarios en el estado de cosas?

Oscilamos por momentos entre distintas posiciones. Por un lado, sentimos que vamos «a contramano» de las tendencias dominantes: promoción de la participación vs vacío de los espacios; fortalecimiento de las redes sociales vs ruptura de lazos de solidaridad; impulso de lo colectivo vs individualismo; construcción de proyectos comunitarios vs «salvese quien pueda!». Otras veces creemos ubicarnos del otro lado del péndulo y el temor a ser capturados por propuestas que no hacen más que pretender cauterizar las heridas abiertas en el tejido social.

Y por último, ¿cuál es el impacto del contexto social actual en nuestra disciplina y en nosotros mismos como trabajadores de la Psicología? Observamos transformaciones importantes que van desde el agotamiento de las formas tradicionales de intervención, hasta la apertura incipiente de nuevos espacios y modalidades de trabajo. Nosotros mismos como sujetos sociales integrantes de las tan debilitadas capas medias, estamos siendo afectados por esta realidad, y el riesgo de la desafiliación social nos compromete.

Poner en juego nuestra implicación, ser capaces de articular espacios de reflexión y análisis, apostar a la producción inserta en una praxis transformadora, asumir los desafíos y alimentar la esperanza, parecen seguir siendo caminos que vale la pena recorrer. 


\section{Bibliografía}

BERGER, P. Y LUCKMANN, T. (1994). La construcción social de la realidad. Bs. As. Amorrortu editores.

Dabas, Elina y Najmanovich, Denise (comp) (1995). Redes El lenguaje de los vinculos. Hacia la reconstrucción y el fortalecimiento de la Sociedad Civil. Bs. As. Paidos.

Fals Borda, O. y Rodriguez Brandao, C. (1986). Investigación Participativa. Instituto del Hombre. Montevideo. Ed Banda Oriental.

Giménez, Luis (comp) (1998). Cruzando Umbrales. Aportes uruguayos en Psicología Comunitaria. Montevideo, Roca Viva.
IbaÑEz Gracia, Tomás (coord). (1989). El conocimiento de la realidad social. Barcelona, Sendai ediciones.

Lapalma, A., Robirosa M. y CARDARELli G.(1990). Turbulencia y planificación social. Bs. As. Unicef, Siglo XXI.

Max NeEF, MANFred y cols. (1986) Desarrollo a escala humana. Montevideo, Nordan.

Montero, Maritza (1991) Concientización, conversión y desideologización en el trabajo psicosocial comunitario. Boletín de la AVEPSO, Vol XIV, N $\square 1$. Venezuela.

RebelLAto, José L. y GiméneZ, L. (1997) Etica de la autonomía. Montevideo.Roca Viva. 